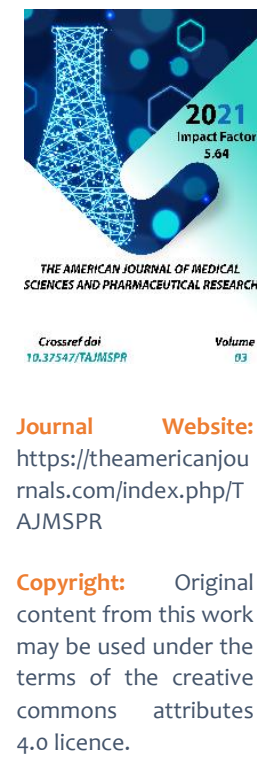

\title{
Social And Medical Aspects Of Left Ventricular Diastolic Dysfunction In Arterial Hypertension
}

Baratova Mehribon Subidinovna

Associate Professor, Department Of Public Health Management, Bukhara State Medical Institute, Bukhara, Uzbekistan

\section{ABSTRACT}

Chronic heart failure (CHF) is a syndrome of several chronic diseases of the cardiovascular system. Among them, arterial hypertension (AH) occupies an important place. High blood pressure (BP) is considered to be the most common cause of CHF. The progression of hypertension towards heart failure includes geometric changes in LV and DD.

\section{KEYWORDS}

Chronic heart failure, hypertension, left ventricular, diastolic dysfunction.

\section{INTRODUCTION}

In heart disease, the main indicator that determines its outcome is the degree of heart muscle dysfunction, which underlies the heart failure syndrome. According to epidemiological study EPOHA-CSF, the prevalence of clinically significant chronic heart failure (CHF) in Russian population is $5.5 \%$, which is 3-10 times higher than in the West. However, if we take into account patients with asymptomatic left ventricular dysfunction, we can speak about $11.7 \%$ of the population [1]. CHF increases the risk of mortality by a factor of 4 , the annual mortality rate of patients ranges from 15 to $50 \%$, and it is CHD patients with left ventricular dysfunction that have the worst prognosis of survival $[2,3]$.

The continuing rise in the prevalence of $\mathrm{CHF}$ is indicative of the inadequate assessment of the functional status of cardiac patients in medication-assisted correction. The progression of cardiovascular disease, from risk factors to the development of fatal complications, is associated with an increase in the activity of the renin-angiotensin- 
aldosterone system (RAAS) [4,5]. Therefore, RAAS blockade is pathogenetically justified and promising, and the ability of the drug to exert additional organ protective effects can be considered as one of the decisive conditions for the choice of treatment. Therefore, the physician is often faced with the problem of choice in the treatment of patients with CHD with left ventricular dysfunction. Which class of drugs that inhibit RAAS activity should be preferred in a particular clinical situation? There is no unequivocal answer to this question today. The indications for angiotensinconverting enzyme inhibitors (ACEIs) and angiotensin II receptor antagonists (ARAs) are identical $(2,6,7)$. Both classes of medicines reduce blood pressure (BP), slow the progression of chronic heart failure (CHF) and protect target organs. However, in practice, iAPPs are used much more frequently than ARAs, as this class of medicines is better understood, more familiar to clinicians and more accessible to patients.

\section{MATERIALS AND METHODS}

Arterial hypertension $(\mathrm{AH})$ is currently one of the most significant medical and social problems. This is due both to the high prevalence of this disease (about $30-45 \%$ of the adult population has high blood pressure (BP)) and to the fact that $\mathrm{AH}$ is a major risk factor for major cardiovascular diseases [3,4]. Along with abdominal obesity, carbohydrate and lipid metabolism disorders, $\mathrm{AH}$ is included in the concept of metabolic syndrome (MS). $\mathrm{AH}$ is accompanied by a higher frequency of dyslipidemia detection, as well as more pronounced metabolic changes [8, 12]. The relevance of studying $M S$ is related to its high prevalence and risk of cardiovascular complications and premature death [11, 13]. According to the International Diabetes
Federation, approximately $20-25 \%$ of the global adult population has MS, with a steadily increasing incidence among children and adolescents due to the increasing prevalence of obesity [9]. Globally, according to data published in 2016 by the World Health Organization, more than 1.9 billion adults over 18 years of age were overweight, of whom more than 650 million were obese. In Russia, 23.5 million obese people were registered at the end of 2016 [10]. People with MS have a 2-3 times higher risk of developing myocardial infarction (MI) and a 5 times higher risk of developing type 2 diabetes mellitus (T2DM) [1214,18-20]. Early detection, treatment and prevention of MS is an important medical and social problem in modern healthcare [15]. The identification of MS is clinically important because, on the one hand, this condition is reversible: with appropriate and timely treatment, the disappearance or at least reduction of the severity of its main manifestations can be achieved [9]. On the other hand, MS precedes the occurrence of such diseases as type $26 \mathrm{DM}$, coronary heart disease (CHD), chronic heart failure (CHF) of ischemic genesis [11]. The pathogenesis of MS is currently being extensively studied, but it has not been fully elucidated. The mechanism of vascular and cardiac damage in MS is of particular interest, as this pathology is the main one in terms of its impact on quality of life and life expectancy in individuals with MS $[2,4]$. According to several studies, MS is closely associated with the development of cardiovascular disorders such as cardiac and vascular wall remodelling and heart failure [5]. A relatively understudied aspect of great interest is the involvement of proinflammatory cytokines in the development of MS. Recently, the inflammatory and regulatory mechanisms involved in the onset and 
progression of MS have been actively studied. Numerous studies have identified an association between MS and its various components and levels of circulating inflammatory markers [3,12]. Several foreign and domestic works indicate the involvement of inflammation mechanisms in the pathogenesis of heart failure, diabetes mellitus, coronary heart disease, abnormal heart geometry and vascular changes $[1,7$,$] . At$ the same time, there is insufficient information available to reveal the pathogenesis of diastolic dysfunction from the perspective of the effect of proinflammatory cytokines on cardiac and vascular remodelling processes in MS. The progression of hypertension towards heart failure includes myocardial fibrosis and changes in left ventricular (LV) geometry. In the presence of these abnormalities, diastolic abnormalities occur, which are defined as LV diastolic dysfunction (HD). These include changes in relaxation and filling, which precede changes in systolic chamber function and can cause symptoms of heart failure even when the ejection fraction is normal. The prevalence of heart failure with normal ejection fraction (HFNEF) has increased over time, whereas the mortality rate from this disorder has remained unchanged. From this perspective, the diagnosis, prognosis and therapeutic management of DD and HFNEF in patients with hypertension represent a growing public health challenge. DD can be asymptomatic and is sometimes detected during Doppler echocardiography. Thus, this tool has gained an important clinical relevance for the diagnosis of DD. A comprehensive assessment of diastolic function should not be performed by simply classifying the progression of $\mathrm{DD}$, but by assessing the degree of LV filling pressure (FP), a true determinant of symptoms and prognosis. This can be obtained by various ultrasound manoeuvres/instruments, but the ratio between transmitter E-rate and early diastolic rate derived from pulsed tissue Doppler (E/e ' ratio) is the most feasible and accurate. Detection of left atrial enlargement may be useful in uncertain cases. The recommended treatment of $\mathrm{DD}$ in patients with arterial hypertension should be consistent with lowering blood pressure (BP) and attempting to reduce LV mass and normalise LV geometry. A comprehensive assessment of DD should not be performed simply by classifying the progression of $\mathrm{DD}$, but by assessing the extent of LV FP, the true determinant of symptoms and prognosis. The practitioner's approach to the hypertensive patient with DD should be consistent with a reduction in $\mathrm{BP}$ and an attempt to reduce $\mathrm{LV}$ mass and normalise LV geometry. Prospective studies with well-defined inclusion criteria ('true' normal PV, concentric LV geometry, standardised assessment of LV diastolic function) are needed to establish whether this approach can reflect a better prognosis in patients with arterial hypertension. Pulmonary hypertension (PH) is commonly seen in patients with left ventricular diastolic dysfunction (LVDD) and is considered a marker of poor prognosis. Although LH in this setting is thought to result from congestion in the pulmonary veins, there is a subgroup of patients whose pulmonary pressures do not improve with appropriate management of diastolic heart failure and who develop a clinical picture similar to that of patients with pulmonary arterial disease. hypertension (LAH). Although Doppler echocardiography and stress tests are useful in the initial assessment of patients with suspected $\mathrm{PH}$ LVDD, the diagnosis can only be confirmed by right heart catheterisation. The management of PH-LVDD is aimed at both optimising fluid 
diversion and reducing post lead to reduce left ventricular diastolic pressure, as well as increasing pulmonary vein return. To date, there is no clear evidence that the addition of LV-specific drugs can improve clinical outcomes and their use should only be considered in the context of clinical trials. The progression of hypertensive left ventricular (LV) lesions towards heart failure involve serial structural abnormalities (mainly myocardial fibrosis) and geometric changes of the left ventricle - concentric LV remodelling and LV hypertrophy (LVH) with a high LV mass/volume ratio, which is a prognostic sign. In the presence of these abnormalities, there are concomitant abnormalities in the diastolic properties of the LV. These abnormalities are broadly defined as LV diastolic dysfunction (LD). They include changes in both relaxation and filling, precede changes in LV systolic chamber function and may themselves cause symptoms/signs of heart failure even when the ejection fraction (EF) is normal (heart failure with normal ejection fraction (EF). Notably, compared with patients with reduced systolic function, patients with HFNEF are more likely to be female, older and less prone to coronary artery disease and more prone to hypertension. The growing interest in DD and HFNEF is driven by the knowledge that $~ 60 \%$ of patients with heart failure have normal or nearnormal EF. 7 The prevalence of HFNEF has increased over the past 15 years - perhaps also reflected in the greater number of patients undergoing Doppler echocardiographic examination - while the mortality rate from this disorder has remained unchanged. 8 Accordingly, the diagnosis, prognosis and therapeutic management of DD and HFNEF in patients with hypertension is a growing public health problem[ 3,6$]$.
Physiology of diastole. The traditional

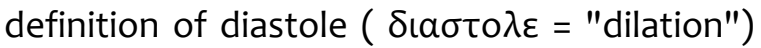
includes the part of the cardiac cycle that begins at aortic valve closure - when LV pressure falls below aortic pressure - and ends at mitral valve closure. Normal diastolic function is clinically defined as the ability of the left ventricle to accept filling volume and its ability to guarantee adequate stroke volume during low-pressure operation. Descriptively, diastole can be divided into four phases:

- Isovolumetric relaxation: this is the period between the end of systolic ejection (aortic valve closure) and the opening of the mitral valve when LV pressure continues to fall rapidly and LV volume remains constant[7].

- Rapid filling of the LV: starts when LV pressure falls below left atrial (LV) pressure and the mitral valve opens. During this period, the blood has an acceleration that reaches a maximum degree directly related to the atrioventricular pressure value and stops when this gradient ends. This period represents a complex interaction between LV suction (active relaxation) and the viscoelastic properties of the myocardium (pliability)[8].

- Diastasis: this occurs when LV and LV pressures are almost equal, and LV filling is mainly maintained by the flow coming from the pulmonary veins - the left atrium is a passive channel - in an amount depending on LV pressure, a function of the 'pliability' of the LV[15].

- Atrial systole: corresponds to LV contraction and ends with mitral valve closure. This period depends on LV compliance and, to a lesser extent, on pericardial resistance, atrial force and atrioventricular synchrony (PR interval obtained on electrocardiogram). Globally, 
LV filling is determined by the interaction between LV filling pressure (LVFP) and filling properties, which in turn are regulated by external factors (mainly pericardial restriction and ventricular interaction) and internal factors such as chamber stiffness (cardiomyocytes and extracellular matrix). myocardial tone, chamber geometry and wall thickness. Increased LVFP is the main physiopathological consequence of DD. It is determined by LV wall filling and passivity and may be additionally controlled by incomplete LV relaxation and altered diastolic myocardial tone. The main morphological and functional correlates of DD include concentric LV geometry, LV enlargement and function, and pulmonary arterial hypertension12,16].

\section{CONCLUSIONS}

Thus, the prevalence of $\mathrm{CHF}$ in patients with $\mathrm{AH}$ and $\mathrm{CHD}$ shows an increase in time (ageing population, increasing proportion of comorbid patients). According to the results of the presented study, the prevalence of CHF was $70 \%$. Unfortunately, the objectives of this study, in particular, the assessment of inhospital treatment of patients with $\mathrm{CHF}$ according to the current guidelines for $\mathrm{CHF}$, could not be fully achieved. The reason for this is that a rather large number (84\%) of patients with CHFsFV have been identified, whereas the clinical guidelines focus primarily on patients with CHFsFV. This, in turn, requires the expansion of diagnostic measures in accordance with the guidelines (in this study, the main diagnostic method was twodimensional EchoCG with Doppler analysis), as well as further study of the disease and determination of treatment strategies for patients with CHFNSFV.

\section{REFERENCES}

1. F.T. Ageev [et al]. (2004). Patients with chronic heart failure in Russian outpatient practice: peculiarities of contingent, diagnostics and treatment (on materials of EPOHA - O - CHF study). Journal of Heart Failure. 5(1). 4 7.

2. F.T. Ageev [et al.]. (2010). Chronic heart failure. Moscow: GOETAR-Media. P.336.

3. Y.N. Belenkov [et al.]. (2003). The first results of national epidemiological study - epidemiological investigation of CHF patients in real clinical practice (by referral) - EPOHA - O - CHF. Journ. cardiac insufficiency. 4(3). pp.116-121.

4. Kulikov A.N. (2003). Opportunities of optimization of diagnostics and treatment of hypertensive disease based on daily monitoring and autometry of arterial pressure: abstract of Ph. D. in Medicine. SPb. VMedA. P.44.

5. I.A. Litovsky [et al.]. (2011). Hypertensive disease - a myth or reality? (Continued). Novye SanktPeterburgskie vedomosti. No.2. pp.7983.

6. Malov, Y.S. (2011). Using principle of "gold proportion" for diagnostics of chronic cardiac insufficiency degree. Bulletin of Russian Military Medical Academy. 2(34). pp.101-105.

7. Ilkhomovna, K. M., Eriyigitovich, I. S., \& Kadyrovich, K. N. (2020). Morphological Features of Microvascular Tissue Of The Brain At Hemorrhagic Stroke. The American Journal of Medical Sciences and Pharmaceutical Research, 2(10), 53-59. 
8. M. I. Kamalova, N.K.Khaidarov, Sh.E.Islamov, (2020). Pathomorphological Features of hemorrhagic brain strokes. Journal of Biomedicine and Practice, Special issue, pp. 101-105.

9. Kadyrovich, K. N., Erkinovich, S. K., \& Ilhomovna, K. M. (2021). Microscopic Examination Of Postcapillary Cerebral Venues In Hemorrhagic Stroke. The American Journal of Medical Sciences and Pharmaceutical Research, 3(08), 913.

10. Shomurodov K.E. (2010). Peculiarities of cytokine balance in gingival fluid at odontogenicphlegmon of the maxillofacial area. The doctor-aspirant. 42(5.1). pp.187-192.

11. Isomov M.M., Shomurodov K.E. (2020). Peculiarities of rehabilitation of pregnant women with inflammatory diseases of the maxillofacial area. International scientific-practical conference "Modern aspects of complex dental rehabilitation of patients with maxillofacial defects". Krasnodar. pp.72-76.

12. Shulutko B.I. (2010). Rational Therapy of Arterial Hypertension. Novie SanktPeterburgskie Vedomosti. No.4. pp.4854.

13. Burnier, M. (1994). Renal sodium handing in patients with untreated hypertension and with coat hypertension. Hypertension. 23(4). pp. 496-502.

14. Effects of treatment on morbidity in hypertension, II: results in patients with diastolic blood pressure averaging 90 through $114 \mathrm{~mm} \mathrm{Hg}$. JAMA. 1970. 213 (7). pp. 143-152.
15. Fuster, V. (2006). ASS/ANA/ ESC 2006 guidelines for the management of patients with an atrial fibrillationexecutive summary: a report of the American College of Cardiology. American Heart Association Task Force on practice guidelines and the European Society of Cardiology Committee for Practice Guidelines (Writing Committee to Revise the 2001 Guidelines for the Management of Patients with Atrial Fibrillation). Eur. Heart J. 27(16). pp.1979-2030.

16. Habbal [et al.]. (1998). Frequence des diagnostics abusifs de I'Hypertension arterielle. Arch. Mal. Coeur Vaiss. 91(8). pp.971- 974 .

17. Hoegholm, A. (1993). Left ventricular mass and geometry in patients with established hypertension and white coat hypertension. Am. J. Hypertens. 6(4). pp.282-286.

18. Murodova, M. M., Baratova, M. S., Fayzullaev, T. T., \& Shagiyazova, L. M. (2020). Diagnostics of functional changes of the left ventricle leading to disorders of the heart rhythm. Central Asian Journal of Pediatrics, 2020(4), 4452.

19. Baratova, M. S. (2021). Algorithm and ultrasonic indicators of the standing of the left atrial in diastolic dysfunction of the left ventricular.

20. Ataeva, M. A., Jarylkasynova, G. J., \& Baratova, M. S. (2020). Assessment of heart rhythm disorders at left atrial stunning at early stages of left ventricular modelling. Journal of Critical Reviews, 7(4), 1695-1699.

21. Ramazanov, M. A., Huseynova, A. S., Hajiyeva, F. V., \& Atayeva, S. U. (2020). Influence of Electrothermopolarization 
The American Journal of Medical Sciences and Pharmaceutical Research

(ISSN - 2689-1026)

Published: September 24, 2021 | Pages: 14-20

IMPACT FACTOR

2021: 5.64

Doi: https://doi.org/10.37547/TAJMSPR/Volume03Issue09-04

OCLC - 1121105510

on $\quad \mathrm{PE}+\quad \mathrm{PbCrO} 4-$ Based

Nanocomposition Structures.

Integrated Ferroelectrics, 211(1), 160-

166.

22. Ataeva, M. A., Jarylkasynova, G. J., \&

Baratova, M. S. (2020). Assessment of

heart rhythm disorders at left atrial

stunning at early stages of left

ventricular modelling. Journal of

Critical Reviews, 7(4), 1695-1699. 